\title{
Nitric Oxide Manipulation: A Therapeutic Target for Peripheral Arterial Disease?
}

\author{
Gareth Williams, ${ }^{1}$ Xu Shi-Wen, ${ }^{2}$ David Abraham, ${ }^{2}$ Sadasivam Selvakumar, ${ }^{3}$ \\ Daryll M. Baker, ${ }^{1}$ and Janice C. S. Tsui ${ }^{1}$ \\ ${ }^{1}$ Division of Surgery \& Interventional Science, University College London, Royal Free Campus, London NW3 2QG, UK \\ ${ }^{2}$ Centre for Rheumatology \& Connective Tissue Disease, University College London, Royal Free Campus, London NW3 2QG, UK \\ ${ }^{3}$ Department Of Surgery, Lister Hospital, Coreys Mill Lane, Stevenage, Hertfordshire SG1 4AB, UK
}

Correspondence should be addressed to Janice C. S. Tsui, janice.tsui@ucl.ac.uk

Received 15 July 2011; Accepted 16 December 2011

Academic Editor: Sidney G. Shaw

Copyright ( $) 2012$ Gareth Williams et al. This is an open access article distributed under the Creative Commons Attribution License, which permits unrestricted use, distribution, and reproduction in any medium, provided the original work is properly cited.

Peripheral Arterial Disease (PAD) is a cause of significant morbidity and mortality in the Western world. Risk factor modification and endovascular and surgical revascularisation are the main treatment options at present. However, a significant number of patients still require major amputation. There is evidence that nitric oxide (NO) and its endogenous inhibitor asymmetric dimethylarginine (ADMA) play significant roles in the pathophysiology of PAD. This paper reviews experimental work implicating the ADMA-DDAH-NO pathway in PAD, focussing on both the vascular dysfunction and effects within the ischaemic muscle, and examines the potential of manipulating this pathway as a novel adjunct therapy in PAD.

\section{Introduction}

Peripheral arterial disease (PAD) is a disorder characterised by the progressive occlusion of the large- and medium-sized arteries outside of the cardiopulmonary and cerebral vascular systems. It causes considerable morbidity and mortality, affecting over 2.7 million individuals in the Western world [1] and most commonly manifests itself in the reduction of blood flow to the lower limbs, which in severe cases lead to chronic pain, gangrene, and eventually limb loss. Risk factors include tobacco smoke, diabetes mellitus, hypertension, and hypercholesterolaemia.

At a molecular level, dysfunction of the vascular endothelium, in particular, disruption of the nitric oxide (NO) pathway [2], plays a significant role. $\mathrm{NO}$ is a vasodilator synthesized by the enzyme nitric oxide synthase (NOS) [3] and has a number of important endocrine and paracrine effects, including reduction of vascular smooth muscle tone; inhibition of platelet adhesion and aggregation; suppression of inflammatory mediators; inhibition of smooth muscle proliferation and migration; and promotion of endothelial survival and repair [4].
There have been significant efforts to elucidate the pathophysiological role of the NO pathway in cardiovascular diseases, with recent discoveries of endogenous NO inhibitors adding further complexity [5], but this has yet to be translated into clinically significant therapies.

This paper aims to outline the current knowledge on NO pathway dysfunction in PAD, including recent developments in understanding the role of asymmetric dimethylarginine (ADMA) and dimethylarginine dimethylaminohydrolase (DDAH); it will also explore potential therapeutic strategies based on the manipulation of this pathway.

\section{The Nitric Oxide Pathway}

NO is synthesised from L-arginine by NOS, of which there are three isoforms [6]: NOS I or neuronal NOS (nNOS) was originally isolated from rat and porcine cerebellum; NOS II or inducible NOS (iNOS) from activated macrophages; NOS III or endothelial NOS (eNOS) from endothelial cells.

All 3 NOS isoforms are competitively inhibited by the methylarginines ADMA and monomethyl-arginine (LNMMA) which are by-products of protein degradation [7]. 


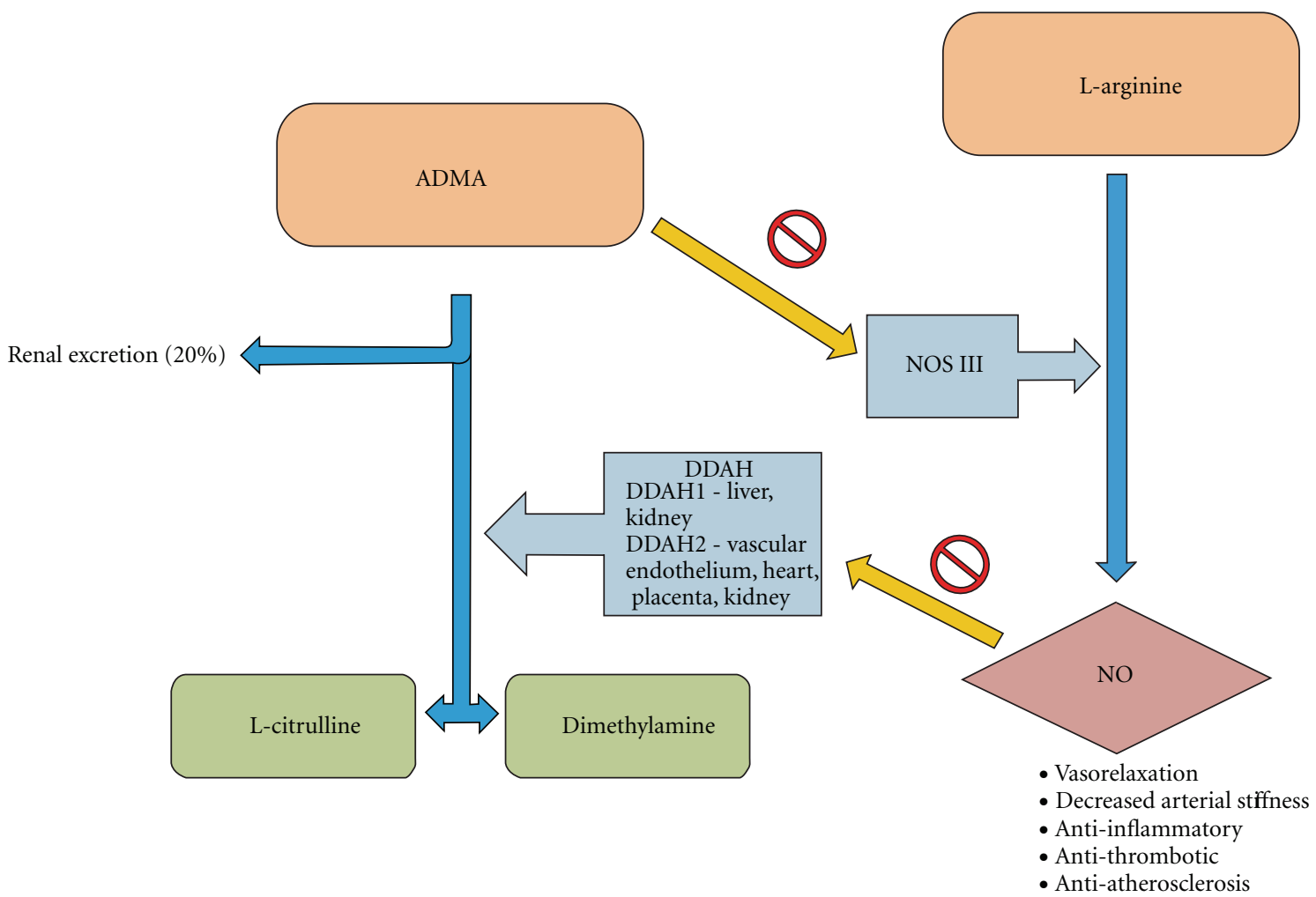

FIgURE 1: The nitric oxide pathway.

Both ADMA and L-NMMA are predominantly broken down by the enzyme DDAH into L-citrulline and dimethylamine [8], of which there are two isoforms [9]: DDAH I, found predominantly in tissues expressing NOS I, liver, kidney, and lung; and DDAH II, found in tissues expressing NOS III and NOS II, vascular endothelium, heart, placenta, and kidney. Both isoforms have been found to be expressed in vascular endothelium [9] (Figure 1).

ADMA's role in vascular endothelial dysfunction was first described by Vallance et al. in patients with end-stage chronic renal failure, whose serum ADMA is raised due, in part, to decreased renal clearance [10]. Further studies in similar patients have shown a positive correlation between ADMA levels and cardiovascular morbidity and mortality [11]; whilst reduction of ADMA levels by renal dialysis helps to restore endothelial function [12].

Other studies have demonstrated a correlation between ADMA accumulation and cardiovascular risk as well as other disease states [5]. Lu et al. showed that serum ADMA levels have a positive correlation with the severity and extent of coronary artery atherosclerosis [13]; Worthmann et al. demonstrated a similar correlation between ADMA levels and adverse prognosis in ischaemic stroke [14]; and ADMA has been found to have significant roles in renal disease [15], pulmonary artery hypertension [16], and erectile dysfunction [17].

The accumulation of ADMA and consequent endothelial dysfunction, increased systemic vascular resistance, and increased systemic blood pressure have been shown to be due to reduced DDAH expression [18]. Upregulation of DDAH I and DDAH II in human umbilical vein endothelial cells (HUVECs) by adenoviral vectors leads to a fall in ADMA and an increase in NO [7]. Similarly, upregulation of DDAH I and II in DDAH I ${ }^{+/-}$mice was found to attenuate the cardiovascular stress response, enhance acetylcholinemediated relaxation, and counteract the response to excess ADMA; DDAH upregulation helps to reverse the cardiovascular dysfunction inherented in these animals [7].

However, DDAH I appears to be the main isoform responsible for ADMA metabolism: DDAH I knockout mice demonstrated significantly raised ADMA levels and systolic blood pressure compared to wild types, despite DDAH II expression remaining normal [19].

These studies suggest that DDAH manipulation may be a useful tool in the treatment of cardiovascular disease.

\section{Peripheral Arterial Disease (PAD)}

In $\mathrm{PAD}$, lower limb ischaemia results in intermittent claudication: this describes pain in the affected muscle groups brought on by exercise and relieved by resting. In more severe PAD, critical limb ischaemia (CLI) occurs: the patient experiences chronic pain at rest, with blood flow to the limb compromised to the extent that the affected limb is at risk, with development of ulcers and gangrene.

The Fontaine classification [20] stratifies these symptoms to correlate with severity of the disease (Table 1 ). 
TABLE 1: Fontaine Classification: stages III and IV are also known as CLI.

\begin{tabular}{cc}
\hline Stage I & Asymptomatic \\
\hline Stage II & Intermittent claudication, no rest pain \\
IIa & When walking a distance of greater than $200 \mathrm{~m}$ \\
IIb & When walking a distance of less than $200 \mathrm{~m}$ \\
Stage III & Nocturnal pain and/or pain at rest \\
Stage IV & Tissue loss: ischaemic ulcers and/or gangrene \\
\hline
\end{tabular}

Current therapies for CLI centre on restoring blood flow to the affected limb by surgical and/or endovascular procedures. Unfortunately, of all patients presenting with CLI, only 50\% will have disease amenable to revascularisation with $25 \%$ requiring a primary amputation. The overall prognosis of these patients is also poor: within a year of presentation, a quarter of the patients would have died, 30\% would have had a major amputation, and $20 \%$ continue to suffer from CLI [1].

There is evidence that the NO-ADMA-DDAH pathway plays an important role in PAD. Evidence of systemically impaired NO synthesis has been described by Böger et al. who showed urinary nitrate and cGMP excretion to diminish in PAD patients as the disease progressed [2]. This group also demonstrated that intravenous L-arginine administration induced NO-mediated vasodilation, increasing femoral blood flow in patients with CLI, resulting in improved walking distances and symptom scores [21]. Furthermore, raised plasma ADMA levels have also been shown to correlate with disease severity in PAD patients [5], implicating the involvement of ADMA.

NO also promotes angiogenesis, activating vascular endothelial growth factor and fibroblast growth factor [22]. Administration of $\mathrm{NO}$ antagonists to canine coronary ischaemia models has demonstrated that NO is an important mediator of new vessel growth [23]. This mechanism of stimulation of new blood vessel development to supply ischaemic tissue has been proposed as a possible treatment for PAD, but clinical results have so far been disappointing. The formation and persistence of new blood vessels is likely to be multifactorial, involving a complex interaction between chemokines and mechanical forces [24]. In addition, continued exposure to cardiovascular risk factors, such as hypercholesterolaemia and hyperglycaemia, has been shown to have a deleterious effect on the NO pathway $[25,26]$.

However, the NO-ADMA-DDAH pathway role in PAD is not confined to vascular dysfunction: NO-ADMA-DDAH interaction is known to play significant parts in the function, homeostasis, and repair of skeletal muscle. Weight for weight, this is the most abundant tissue in the lower limb and the majority of PAD symptoms are attributable to skeletal muscle ischaemia; it can almost be regarded as a "target organ" of PAD. Hence, the NO-ADMA-DDAH pathway within ischaemic skeletal muscle represents a significant facet of potential CLI therapy.

\section{NO in Skeletal Muscle}

Skeletal muscle has been shown to express both NOS I and NOS III: NOS I is localized to the sarcolemma, and NOS III associated with mitochondria and microvessels within the tissue [27]. Within skeletal muscle, NO acts as an endogenous modulator of vascular tone $[28,29]$, neuromuscular transmission [30], muscle contraction [31, 32], muscle metabolism [27] and myogenesis [33]. Its role in the regulation of muscle contraction is complex and is influenced by the muscle fibre type and contraction pattern and frequency involved [31]. NO has a number of effects on skeletal muscle metabolism [27]. It affects glucose metabolism, upregulating glucose uptake and inhibiting glycolysis [34]; it has a direct inhibitory effect on mitochondrial respiration [35]; and it antagonises creatinine kinase activity, decreasing phosphocreatine breakdown, limiting muscle function [36].

NO plays a role in myogenesis as well as repair and regeneration following injury. It has been shown to modulate the inflammatory response to injured skeletal muscle, controlling blood flow to the injured tissue, and affecting subsequent remodelling and repair [37]. In experimental models of muscle crush injury, upregulation of local NO has been demonstrated [38], inhibiting adhesion and inducing apoptosis in inflammatory cells [39]. NOS is activated in response to stretch in skeletal muscle, mediating the release of hepatocyte growth factor and activating mononuclear progenitor cells called satellite cells (SC), which are responsible for post-injury remodelling [40].

Tidball et al. showed that the addition of exogenous NO upregulated the expression of structural proteins within mouse myotubes and found that NOS antagonists inhibited this, suggesting a regulatory role for NO in myogenesis [33].

In addition to local effects, $\mathrm{NO}$ appears to play a part in the systemic inflammatory response: experimental rats with peritonitis or septic shock have been shown to have increased expression of NOS in their skeletal muscle [41].

However, NO has also been shown to have deleterious effects, reacting with superoxide anions to form harmful nitrogenous intermediates which are directly toxic to skeletal muscle and inhibit mitochondrial function $[41,42]$.

\section{NO in Ischaemia}

Impaired NO-mediated vasodilation has been found in postischaemic tissue such as myocardium [43], brain [44], and skeletal muscle [45]. There is a complex interaction between ischaemia and the NOS isoforms, with both up- and downregulation reported in ischaemic models; for instance, rat brain oxygen-glucose-deprivation models have shown NOS II to be upregulated in response to ischaemia, which in turn causes a downregulation of NOS I [46]; however, ischaemic preconditioning of rat liver has been shown to upregulate NOS III [47].

In skeletal muscle from patients with CLI, we have previously found raised NOS III expression associated with both microvessels within ischaemic muscle sections as well as the muscle fibres themselves [48]. However, this was not 


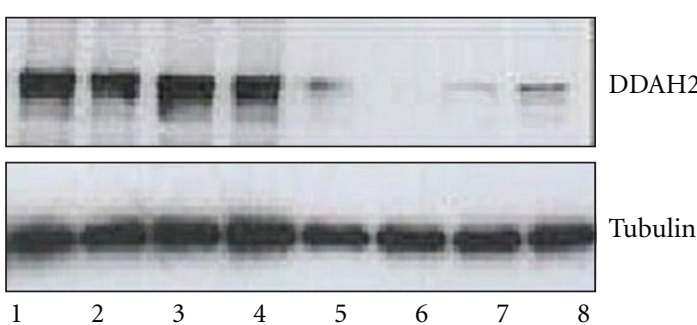

(a)

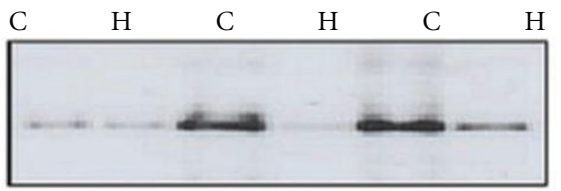

DDAH2

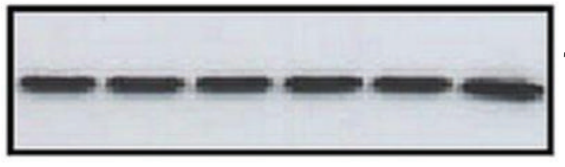

(c)

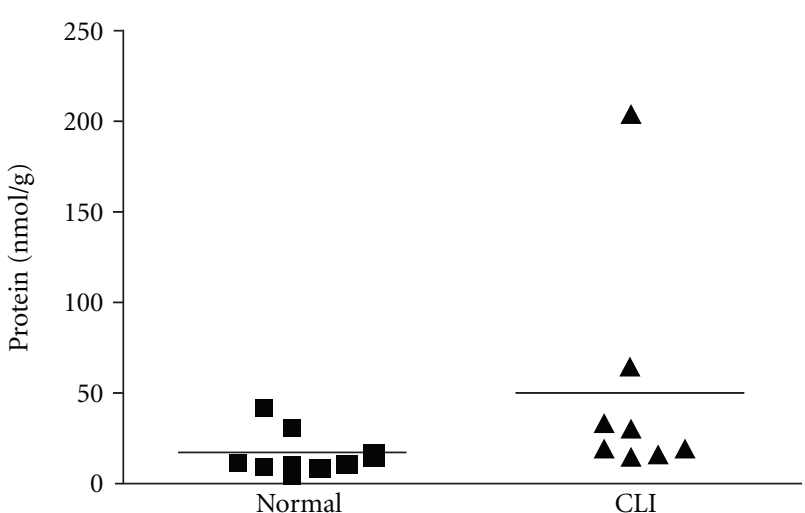

(b)

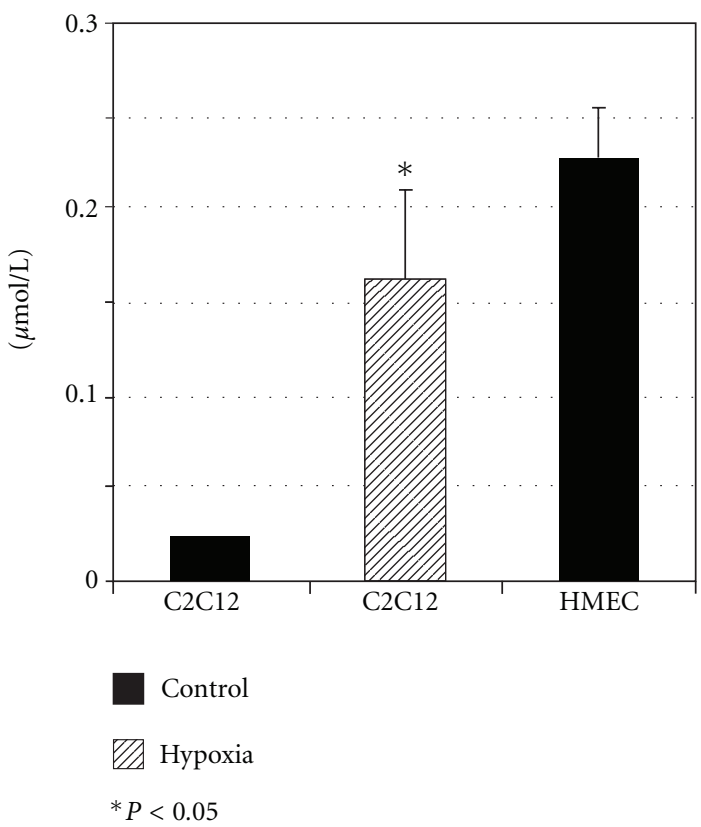

(d)

FIGURE 2: ADMA/DDAH pathway in ischaemic muscle-preliminary data. (a) Western blot showing reduced DDAH2 expression in muscle biopsies from patients with CLI. Lanes 1-4: control, 5-8: CLI muscle. (b) ELISA showing significantly higher ADMA levels in muscle from patients with CLI $(P=0.03$, Mann Whitney $U$ test). (c) Western blot showing reduced DDAH2 expression in hypoxic myotubes (C: control, $\mathrm{H}$ : hypoxia). (d) Conditioned medium from hypoxic C2C12 myotubes contained elevated levels of ADMA compared to medium from myotubes cultured in normoxia. $(P<0.05$, Student's $t$-test). Medium from HMEC-1 cells was used as positive control.

associated with an increase in NOS activity. The involvement of endogenous inhibitors may explain this and recent preliminary data suggesting that ADMA levels in muscle homogenates from patients with CLI are raised (Figures $2(\mathrm{a})$ and 2(b)). In addition, DDAH2 protein levels are also upregulated in these ischaemic muscle biopsies.

To study the potential role of the ADMA/DDAH pathway in skeletal muscle further, we evaluated their levels in myotubes cultured in ischaemic conditions in vitro. We found reduced DDAH2 expression in ischaemic myotubes, whilst ADMA levels were elevated in ischaemic myotube conditioned media (Figures 2(c) and 2(d)).
Whilst the majority of studies have focussed on ADMA associated with the vasculature/endothelial cells, our findings from our in vitro studies suggest that ischaemic myotubes may be a source of ADMA which can then act in a paracrine fashion on surrounding endothelial cells or in an autocrine manner on myotubes themselves with potentially detrimental effects.

Fiedler et al. found that ADMA impairs vascular endothelial growth factor (VEGF) mediated angiogenesis, and that this was prevented by upregulation of DDAH [49]. Increased levels of ADMA produced by ischaemic muscle may prevent angiogenesis in PAD patients and may be a 
factor in the failure of angiogenesis induction as a clinical therapeutic approach.

\section{Manipulation of the ADMA-DDAH Pathway}

Control of NO levels by manipulating the ADMA-DDAHNO pathway represents a significant avenue for potential early PAD treatment.

As L-arginine is the main precursor for $\mathrm{NO}$ production (Figure 1), an increase in the availability of substrate ought to result in an increase in NO. However, there is conflicting evidence as to whether L-arginine supplementation has a clinically significant benefit in cardiovascular disease: Sydow and Münzel [50] showed that L-arginine improved radial artery dilatation, in contrast to Walker et al's findings that vasodilatation was independent of oral L-arginine supplementation [51]. In PAD, the Nitric Oxide in Peripheral Arterial Insufficiency (NO-PAIN) study randomized 133 patients with intermittent claudication to oral L-arginine versus placebo for 6 months. Whilst L-arginine supplementation increased plasma L-arginine levels, there was no improvement in NO availability or vascular function. This was in contrast to earlier, smaller studies of shorter duration demonstrating a beneficial effect of L-arginine in PAD patients [21]. A mechanism of "arginine-tolerance" was proposed, in a similar manner to "nitrate-tolerance", observed in prolonged nitroglycerine use. More disappointingly, whilst both groups in the NO-PAIN study showed improvement in absolute walking distance, the improvement in the Larginine group was significantly less than the placebo group [52].

An alternative strategy to increase NO levels may be to reduce ADMA levels by enhancing DDAH activity or expression. Nebivolol [53], pioglitazone [54], pravastatin [55], and fenofibrate [56] have all been shown to decrease ADMA by upregulating DDAH in vitro. Clinically, Sen et al. demonstrated an increase in circulating $\mathrm{NO}$ and a decrease in ADMA, coupled with improvement in symptoms in patients with ischaemic heart disease treated with the selective beta1adrenergic receptor antagonist nebivolol [47]. Nishiyama et al. found that the statin, pravastatin reduced serum ADMA in patients with cerebrovascular disease [57].

In PAD, upregulation of DDAH at a local level to reduce ADMA levels within the ischaemic muscle may be of benefit in a number of ways: it may improve microvascular function and local tissue perfusion; it may improve the results of therapeutic angiogenesis by reducing the antiangiogenic environment; or it may improve muscle contractile and metabolic function and perhaps reduce tissue damage. In patients with CLI, such strategies may be used as an adjunct to improve the results of revascularisation techniques as well as reduce tissue damage in patients with non-reconstructable disease. They may also be of benefit to patients with earlier disease to prevent disease progression.

\section{Conclusion}

There is considerable evidence that disruption of the NO pathway plays a major role in the pathogenesis of PAD.
ADMA in particular, through its actions on NOS III, has been shown to be an important modulator of NO. Early work suggests that local or systemic manipulation of ADMA, by upregulating DDAH, may prove to be a novel treatment for PAD.

\section{References}

[1] L. Norgren, W. R. Hiatt, J. A. Dormandy, M. R. Nehler, K. A. Harris, and F. G. R. Fowkes, "Inter-society consensus for the management of peripheral arterial disease (TASC II)," Journal of Vascular Surgery, vol. 45, no. 1, pp. S5-S67, 2007.

[2] R. H. Böger, S. M. Bode-Böger, W. Thiele, W. Junker, K. Alexander, and J. C. Frölich, "Biochemical evidence for impaired nitric oxide synthesis in patients with peripheral arterial occlusive disease," Circulation, vol. 95, no. 8, pp. 20682074, 1997.

[3] R. M. J. Palmer, D. S. Ashton, and S. Moncada, "Vascular endothelial cells synthesize nitric oxide from L-arginine," Nature, vol. 333, no. 6174, pp. 664-666, 1988.

[4] J. P. Cooke, "DDAH: a target for vascular therapy?" Vascular Medicine, vol. 15, no. 3, pp. 235-238, 2010.

[5] J. P. Cooke, "Asymmetrical dimethylarginine: the Uber marker?" Circulation, vol. 109, no. 15, pp. 1813-1819, 2004.

[6] U. Forstermann, M. Nakane, W. R. Tracey, and J. S. Pollock, "Isoforms of nitric oxide synthase: functions in the cardiovascular system," European Heart Journal, vol. 14, no. I, pp. 10-15, 1993.

[7] B. Torondel, M. Nandi, P. Kelly, B. Wojciak-Stothard, I. Fleming, and J. Leiper, "Adenoviral-mediated overexpression of DDAH improves vascular tone regulation," Vascular Medicine, vol. 15, no. 3, pp. 205-213, 2010.

[8] T. Teerlink, "ADMA metabolism and clearance," Vascular Medicine, vol. 10, no. 1, pp. S73-S81, 2005.

[9] J. M. Leiper, J. Santa Maria, A. Chubb et al., "Identification of two human dimethylarginine dimethylaminohydrolases with distinct tissue distributions and homology with microbial arginine deiminases," Biochemical Journal, vol. 343, no. 1, pp. 209-214, 1999.

[10] P. Vallance, A. Leone, A. Calver, J. Collier, and S. Moncada, "Accumulation of an endogenous inhibitor of nitric oxide synthesis in chronic renal failure," Lancet, vol. 339, no. 8793, pp. 572-575, 1992.

[11] C. Zoccali, S. M. Bode-Böger, F. Mallamaci et al., "Plasma concentration of asymmetrical dimethylarginine and mortality in patients with end-stage renal disease: a prospective study," Lancet, vol. 358, no. 9299, pp. 2113-2117, 2001.

[12] M. F. Hand, W. G. Haynes, and D. J. Webb, "Hemodialysis and L-arginine, but not $\mathrm{D}$-arginine, correct renal failureassociated endothelial dysfunction," Kidney International, vol. 53, no. 4, pp. 1068-1077, 1998.

[13] T. M. Lu, Y. A. Ding, M. J. Charng, and S. J. Lin, "Asymmetrical dimethylarginine: a novel risk factor for coronary artery disease," Clinical Cardiology, vol. 26, no. 10, pp. 458-464, 2003.

[14] H. Worthmann, S. Chen, J. Martens-Lobenhoffer et al., "High plasma dimethylarginine levels are associated with adverse clinical outcome after stroke," Journal of Atherosclerosis and Thrombosis, vol. 18, no. 9, pp. 753-761, 2011.

[15] J. T. Kielstein, J. C. Frölich, H. Haller, and D. Fliser, "ADMA (asymmetric dimethylarginine): an atherosclerotic disease mediating agent in patients with renal disease?" Nephrology Dialysis Transplantation, vol. 16, no. 9, pp. 1742-1745, 2001. 
[16] J. T. Kielstein, S. M. Bode-Böger, G. Hesse et al., "Asymmetrical dimethylarginine in idiopathic pulmonary arterial hypertension," Arteriosclerosis, Thrombosis, and Vascular Biology, vol. 25, no. 7, pp. 1414-1418, 2005.

[17] R. Maas, E. Schwedhelm, J. Albsmeier, and R. H. Böger, "The pathophysiology of erectile dysfunction related to endothelial dysfunction and mediators of vascular function," Vascular Medicine, vol. 7, no. 3, pp. 213-225, 2002.

[18] J. Leiper, M. Nandi, B. Torondel et al., "Disruption of methylarginine metabolism impairs vascular homeostasis," Nature Medicine, vol. 13, no. 2, pp. 198-203, 2007.

[19] X. Hu, D. Atzler, X. Xu et al., "Dimethylarginine dimethylaminohydrolase-1 is the critical enzyme for degrading the cardiovascular risk factor asymmetrical dimethylarginine," Arteriosclerosis, Thrombosis, and Vascular Biology, vol. 31, no. 7, pp. 1540-1546, 2011.

[20] R. Fontaine, M. Kim, and R. Kieny, "Surgical treatment of peripheral circulation disorders," Helvetica chirurgica acta, vol. 21, no. 5-6, pp. 499-533, 1954.

[21] S. M. Bode-Böger, R. H. Böger, H. Alfke et al., "L-arginine induces nitric oxide-dependent vasodilation in patients with critical limb ischemia: a randomized, controlled study," Circulation, vol. 93, no. 1, pp. 85-90, 1996.

[22] M. Ziche and L. Morbidelli, "Nitric oxide and angiogenesis," Journal of Neuro-Oncology, vol. 50, no. 1-2, pp. 139-148, 2000.

[23] T. Matsunaga, D. C. Warltier, D. W. Weihrauch, M. Moniz, J. Tessmer, and W. M. Chilian, "Ischemia-induced coronary collateral growth is dependent on vascular endothelial growth factor and nitric oxide," Circulation, vol. 102, no. 25, pp. 3098 3103, 2000.

[24] T. T. Rissanen, I. Vajanto, and S. Ylä-Herttuala, "Gene therapy for therapeutic angiogenesis in critically ischaemic lower limb - On the way to the clinic," European Journal of Clinical Investigation, vol. 31, no. 8, pp. 651-666, 2001.

[25] A. Rivard, J. E. Fabre, M. Silver et al., "Age-dependent impairment of angiogenesis," Circulation, vol. 99, no. 1, pp. 111-120, 1999.

[26] A. Ito, P. S. Tsao, S. Adimoolam, M. Kimoto, T. Ogawa, and J. P. Cooke, "Novel mechanism for endothelial dysfunction: dysregulation of dimethylarginine dimethylaminohydrolase," Circulation, vol. 99, no. 24, pp. 3092-3095, 1999.

[27] M. B. Reid, "Role of nitric oxide in skeletal muscle: synthesis, distribution and functional importance," Acta Physiologica Scandinavica, vol. 162, no. 3, pp. 401-409, 1998.

[28] R. C. Hickner, J. S. Fisher, A. A. Ehsani, and W. M. Kohrt, "Role of nitric oxide in skeletal muscle blood flow at rest and during dynamic exercise in humans," American Journal of Physiology, vol. 42, no. 1, pp. H405-H410, 1997.

[29] S. N. A. Hussain, "Activity of nitric oxide synthase in the ventilatory muscle vasculature," Comparative Biochemistry and Physiology, vol. 119, no. 1, pp. 191-201, 1998.

[30] J. Ribera, J. Marsal, A. Casanovas, M. Hukkanen, O. Tarabal, and J. E. Esquerda, "Nitric oxide synthase in rat neuromuscular junctions and in nerve terminals of Torpedo electric organ: its role as regulator of acetylcholine release," Journal of Neuroscience Research, vol. 51, no. 1, pp. 90-102, 1998.

[31] C. L. Murrant, J. C. Frisbee, and J. K. Barclay, "The effect of nitric oxide and endothelin on skeletal muscle contractility changes when stimulation is altered," Canadian Journal of Physiology and Pharmacology, vol. 75, no. 5, pp. 414-422, 1997.

[32] G. Maréchal and P. Gailly, "Effects of nitric oxide on the contraction of skeletal muscle," Cellular and Molecular Life Sciences, vol. 55, no. 8-9, pp. 1088-1102, 1999.
[33] J. G. Tidball, M. J. Spencer, M. Wehling, and E. Lavergne, "Nitric-oxide synthase is a mechanical signal transducer that modulates talin and vinculin expression," Journal of Biological Chemistry, vol. 274, no. 46, pp. 33155-33160, 1999.

[34] T. W. Balon and J. L. Nadler, "Evidence that nitric oxide increases glucose transport in skeletal muscle," Journal of Applied Physiology, vol. 82, no. 1, pp. 359-363, 1997.

[35] L. Kobzik, B. Stringer, J. L. Balligand, M. B. Reid, and J. S. Stamler, "Endothelial type nitric oxide synthase skeletal muscle fibers: mitochondrial relationships," Biochemical and Biophysical Research Communications, vol. 211, no. 2, pp. 375381, 1995.

[36] W. L. Gross, M. I. Bak, J. S. Ingwall et al., "Nitric oxide inhibits creatine kinase and regulates rat heart contractile reserve," Proceedings of the National Academy of Sciences of the United States of America, vol. 93, no. 11, pp. 5604-5609, 1996.

[37] L. I. Filippin, A. J. Moreira, N. P. Marroni, and R. M. Xavier, "Nitric oxide and repair of skeletal muscle injury," Nitric Oxide, vol. 21, no. 3-4, pp. 157-163, 2009.

[38] I. Rubinstein, Z. Abassi, R. Coleman, F. Milman, J. Winaver, and O. S. Better, "Involvement of nitric oxide system in experimental muscle crush injury," Journal of Clinical Investigation, vol. 101, no. 6, pp. 1325-1333, 1998.

[39] J. G. Tidball, "Inflammatory processes in muscle injury and repair," American Journal of Physiology, vol. 288, no. 2, pp. R345-R353, 2005.

[40] R. Tatsumi, A. Hattori, Y. Ikeuchi, J. E. Anderson, and R. E. Allen, "Release of hepatocyte growth factor from mechanically stretched skeletal muscle satellite cells and role of $\mathrm{pH}$ and nitric oxide," Molecular Biology of the Cell, vol. 13, no. 8, pp. 2909-2918, 2002.

[41] H. J. Kaminski and F. H. Andrade, "Nitric oxide: biologic effects on muscle and role in muscle diseases," Neuromuscular Disorders, vol. 11, no. 6-7, pp. 517-524, 2001.

[42] T. M. Dawson, V. L. Dawson, and S. H. Snyder, "A novel neuronal messenger molecule in brain: the free radical, nitric oxide," Annals of Neurology, vol. 32, no. 3, pp. 297-311, 1992.

[43] P. S. Tsao, N. Aoki, D. J. Lefer, G. Johnson, and A. M. Lefer, "Time course of endothelial dysfunction and myocardial injury during myocardial ischemia and reperfusion in the cat," Circulation, vol. 82, no. 4, pp. 1402-1412, 1990.

[44] W. G. Mayhan, S. M. Amundsen, F. M. Faraci, and D. D. Heistad, "Responses of cerebral arteries after ischemia and reperfusion in cats," American Journal of Physiology, vol. 255, no. 4, pp. H879-H883, 1988.

[45] W. C. Sternbergh, R. G. Makhoul, and B. Adelman, "Nitric oxide-mediated, endothelium-dependent vasodilation is selectively attenuated in the postischemic extremity," Surgery, vol. 114, no. 5, pp. 960-967, 1993.

[46] J. De Alba, A. Cárdenas, M. A. Moro et al., "Down-regulation of neuronal nitric oxide synthase by nitric oxide after oxygenglucose deprivation in rat forebrain slices," Journal of Neurochemistry, vol. 72, no. 1, pp. 248-254, 1999.

[47] N. Şen, Y. Tavil, H. Erdamar et al., "Nebivolol therapy improves endothelial function and increases exercise tolerance in patients with cardiac syndrome X," Anadolu Kardiyoloji Dergisi, vol. 9, no. 5, pp. 371-379, 2009.

[48] J. C. S. Tsui, D. M. Baker, S. G. Shaw, and M. R. Dashwood, "Alterations in nitric oxide synthase isoforms in acute lower limb ischemia and reperfusion," Angiology, vol. 58, no. 5, pp. 586-592, 2007.

[49] L. R. Fiedler, T. Bachetti, J. Leiper et al., "The ADMA/ DDAH pathway regulates VEGF-mediated angiogenesis," 
Arteriosclerosis, Thrombosis, and Vascular Biology, vol. 29, no. 12, pp. 2117-2124, 2009.

[50] K. Sydow and T. Münzel, "ADMA and oxidative stress," Atherosclerosis Supplements, vol. 4, no. 4, pp. 41-51, 2003.

[51] H. A. Walker, E. McGing, I. Fisher et al., "Endotheliumdependent vasodilation is independent of the plasma Larginine/ADMA ratio in men with stable angina: lack of effect of oral L-arginine on endothelial function, oxidative stress and exercise performance," Journal of the American College of Cardiology, vol. 38, no. 2, pp. 499-505, 2001.

[52] A. M. Wilson, R. Harada, N. Nair, N. Balasubramanian, and J. P. Cooke, "L-arginine supplementation in peripheral arterial disease: no benefit and possible harm," Circulation, vol. 116, no. 2, pp. 188-195, 2007.

[53] U. Garbin, A. F. Pasini, C. Stranieri, S. Manfro, V. Boccioletti, and L. Cominacini, "Nebivolol reduces asymmetric dimethylarginine in endothelial cells by increasing dimethylarginine dimethylaminohydrolase 2 (DDAH2) expression and activity," Pharmacological Research, vol. 56, no. 6, pp. 515-521, 2007.

[54] S. Wakino, K. Hayashi, S. Tatematsu et al., "Pioglitazone lowers systemic asymmetric dimethylarginine by inducing dimethylarginine dimethylaminohydrolase in rats," Hypertension Research, vol. 28, no. 3, pp. 255-262, 2005.

[55] Q. F. Yin and Y. Xiong, "Pravastatin restores DDAH activity and endothelium-dependent relaxation of rat aorta after exposure to glycated protein," Journal of Cardiovascular Pharmacology, vol. 45, no. 6, pp. 525-532, 2005.

[56] T. L. Yang, M. F. Chen, B. L. Luo, Q. Y. Xie, J. L. Jiang, and Y. J. Li, "Fenofibrate decreases asymmetric dimethylarginine level in cultured endothelial cells by inhibiting NF- $\kappa$ B activity," Naunyn-Schmiedeberg's Archives of Pharmacology, vol. 371, no. 5, pp. 401-407, 2005.

[57] Y. Nishiyama, M. Ueda, T. Otsuka et al., "Statin treatment decreased serum asymmetric dimethylarginine (ADMA) levels in Ischemic stroke patients," Journal of Atherosclerosis and Thrombosis, vol. 18, no. 2, pp. 131-137, 2011. 


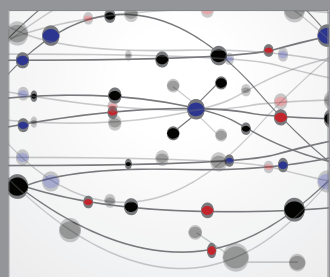

The Scientific World Journal
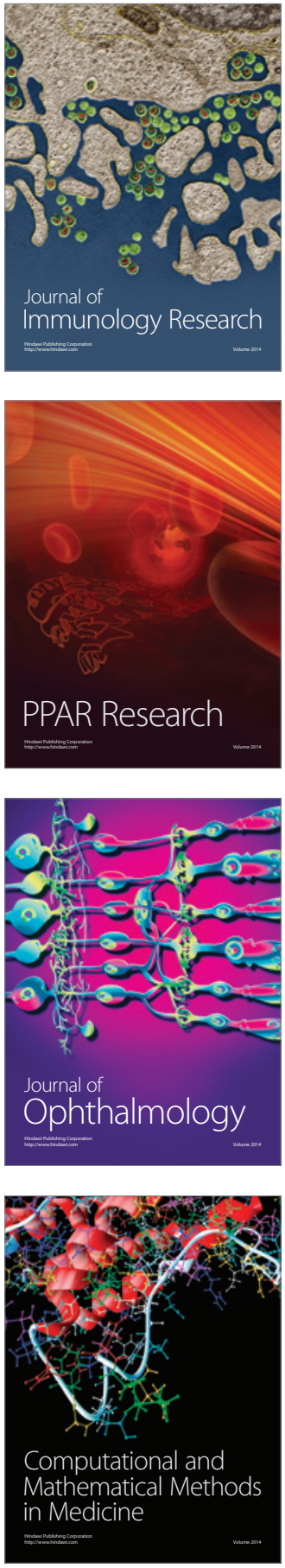

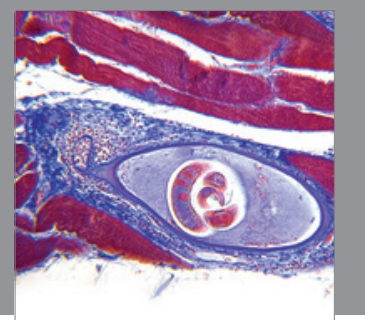

Gastroenterology

Research and Practice
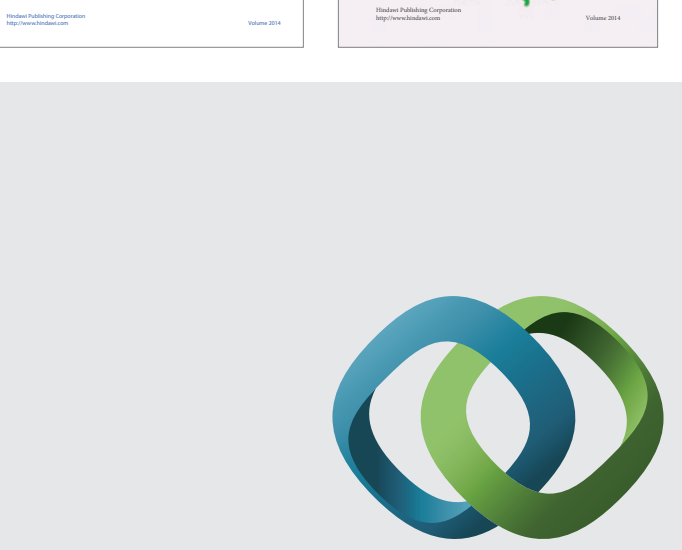

\section{Hindawi}

Submit your manuscripts at

http://www.hindawi.com
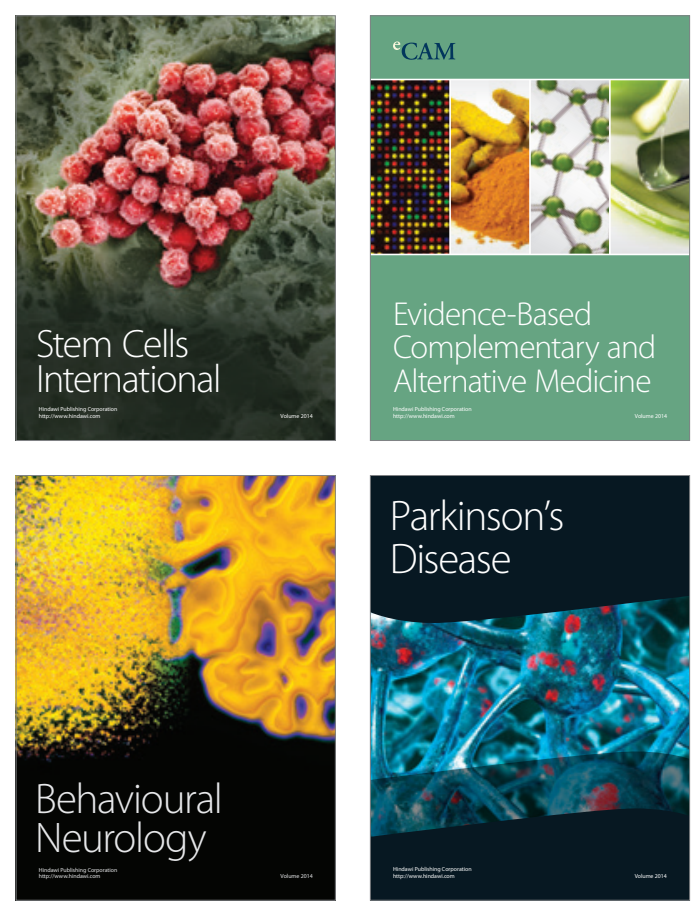

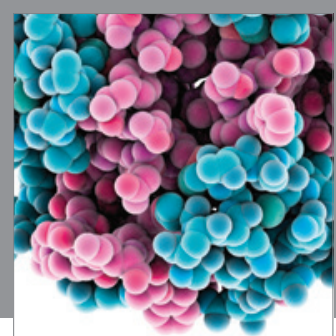

Journal of
Diabetes Research

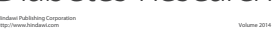

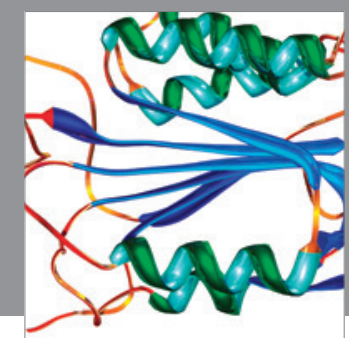

Disease Markers
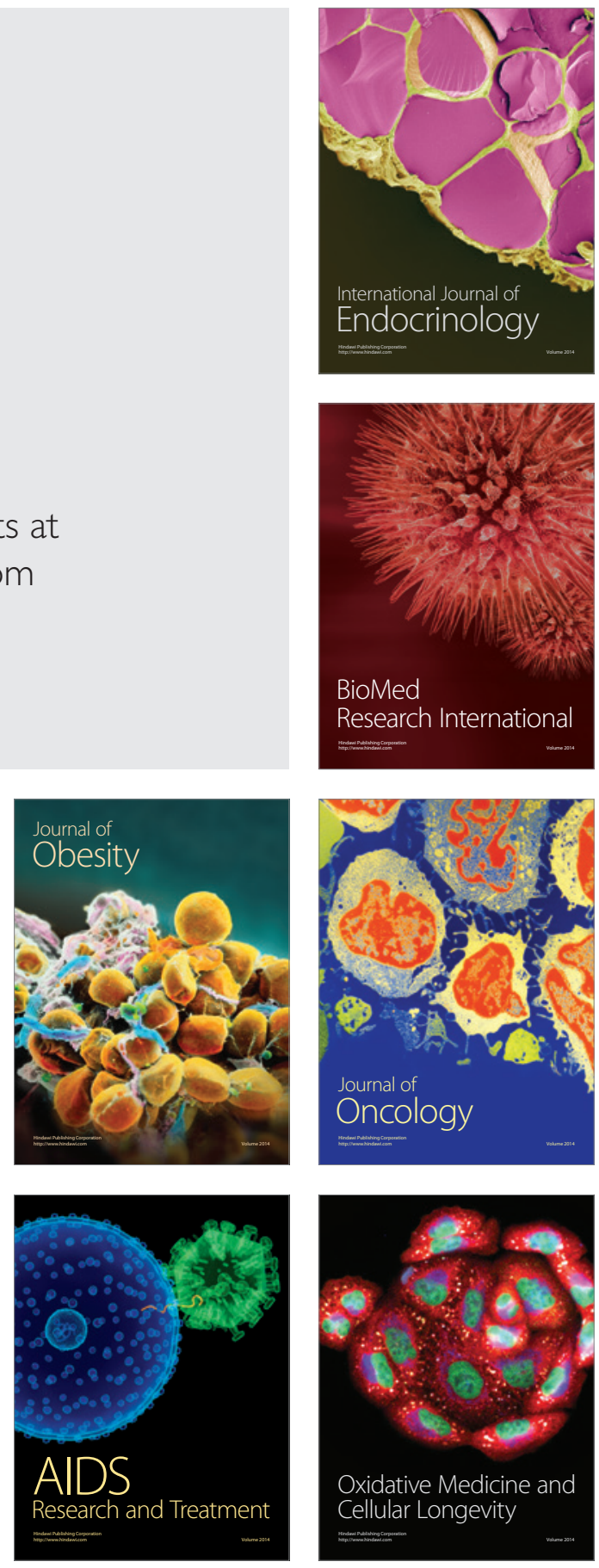